\title{
Cluster Expansion for Abstract Polymer Models
}

\author{
R. Kotecký ${ }^{1}$ and D. Preiss ${ }^{2}$ \\ ${ }^{1}$ Department of Mathematical Physics, Charles University, V Holešovičkách 2, \\ CS-18000 Praha 8, Czechoslovakia \\ ${ }^{2}$ Department of Mathematical Analysis, Charles University, Sokolovská 83, CS-18600 Praha 8, \\ Czechoslovakia
}

\begin{abstract}
A new direct proof of convergence of cluster expansions for polymer (contour) models is given in an abstract setting. It does not rely on KirkwoodSalsburg type equations or "combinatorics of trees." A distinctive feature is that, at all steps, the considered clusters contain every polymer at most once.
\end{abstract}

\section{Introduction}

In the study of classical lattice models it is often useful to rewrite their partition function or their correlations in terms of polymer models. It is so whenever one is able to rewrite the partition function in a volume $V$ in the form

$$
Z(V)=\sum \prod_{\gamma \in \partial} \Phi(\gamma)
$$

where the sum is over all compatible families $\partial$ of certain geometrical objects $(\gamma)$ called polymers. A particular form of the polymer weights $\Phi(\gamma)$ depends on the considered model. See e.g. [1] for a collection of some typical examples. Notice that when applied to low temperature expansions the term contour is used instead of polymer.

If the weights $\Phi(\gamma)$ are small enough for "large" polymers $\gamma$, one may expand $\log Z(V)$ in a convenient form called cluster expansion, enabling one to make good evaluations of the "free energy" ("pressure") $\lim \frac{1}{|V|} \log Z(V)$ as well as of the decay of correlations. There are essentially two approaches to the proof of convergence of cluster expansion: one is based on the use of Kirkwood-Salsburg type of equations [2-5], while the other relies on "combinatorics of trees of a graph" $[1,6,7]$. In both these approaches many copies of one polymer may appear in a cluster. As a consequence the cluster expansion for $\log Z(V)$ is an infinite series even for finite $V$. Our goal here is to present a new formulation and a new proof of cluster expansions which is in our view both simple and straightforward, since it uses, at all stages, only clusters in which every polymer may appear at most once. 
To stress that the formulation as well as the proof do not depend on details of "geometry of polymers" we state our theorem in an abstract setting.

\section{Setting and Results}

We shall consider a countable set $\mathrm{K}$, the elements of which will be called polymers. Let $\imath \subset \mathrm{K} \times \mathrm{K}$ be a reflexive and symmetric relation. A pair $\gamma_{1}, \gamma_{2} \in \mathrm{K}$ is called incompatible (compatible) if $\left(\gamma_{1}, \gamma_{2}\right) \in \imath\left(\left(\gamma_{1}, \gamma_{2}\right) \notin \imath\right)$. We shall also use the notation $\gamma_{1} l \gamma_{2}$ for incompatible polymers. Whenever $L \subset K$, let $\mathscr{B}(L)$ be the family of all finite subsets of $L$ and $\mathscr{D}(L)\left(\mathscr{D}_{0}(\mathrm{~L})\right)$ be the family of all (finite) subsets $\partial C L$ consisting of mutually compatible polymers; $\mathscr{B}=\mathscr{B}(\mathrm{K}), \mathscr{D}=\mathscr{D}(\mathrm{K})$, and $\mathscr{D}_{0}=\mathscr{D}_{0}(\mathrm{~K})$. For $\mathrm{C} \in \mathscr{B}$ we denote by $|\mathrm{C}|$ the number of polymers in $\mathrm{C}$ and write $\mathrm{C} l \gamma$ whenever there exists $\gamma^{\prime} \in \mathrm{C}$ such that $\gamma^{\prime} \imath \gamma$. We call $\mathrm{C} \in \mathscr{B}$ a cluster if it is not decomposable into two nonempty sets, $\mathbf{C}=\mathrm{C}_{1} \cup \mathrm{C}_{2}$, such that every pair $\gamma_{1} \in \mathrm{C}_{1}, \gamma_{2} \in \mathrm{C}_{2}$, is compatible. To evaluate a "decay rate" we shall use a function $d: \mathrm{K} \rightarrow[0, \infty)$, and denote $d(\mathrm{C})=\sum_{\gamma \in C} d(\gamma)$ for every $\mathrm{C} \in \mathscr{B}$.

We suppose the "statistical weights" of polymers to be given by a polymer functional $\Phi: \mathrm{K} \rightarrow \mathbb{C}$, and denote $\Phi(\partial)=\prod_{\gamma \in \delta} \Phi(\gamma)$ for every $\partial \in \mathscr{D}_{0}$ [we put always $\Phi(\emptyset)=1]$. Thus we define the partition function $\mathscr{Z}(\mathrm{L} ; \Phi)$ for every finite $\mathrm{L} \subset \mathrm{K}$ by

$$
\mathscr{Z}(\mathrm{L} ; \Phi)=\sum_{\partial \in \mathscr{D}(\mathrm{L})} \Phi(\partial) .
$$

Whenever $\mathfrak{M}$ is a contractible set of polymer functionals and it is known that $\mathscr{Z}(\mathrm{L} ; \Phi) \neq 0$ for every $\Phi \in \mathfrak{M}$, we may uniquely define $\log \mathscr{Z}(\mathrm{L} ; \Phi)$ as that continuous branch of logarithm for which $\log \mathscr{Z}(\mathrm{L}, \Phi=0)=0$ [let us notice that $\mathscr{Z}(\mathrm{L}, \Phi=0)=1$ for every $L \in \mathscr{B}]$. This is the case (with fixed functions $a$ and $d$ ) in the following theorem and we shall always understand $\log \mathscr{Z}(\mathrm{L} ; \Phi)$ in the above sense.

Theorem. Let functions $a: \mathrm{K} \rightarrow[0, \infty), d: \mathrm{K} \rightarrow[0, \infty)$, and $\Phi: \mathrm{K} \rightarrow \mathbb{C}$ be such that

$$
\sum_{\gamma^{\prime}: \gamma^{\prime} \iota \gamma} e^{a\left(\gamma^{\prime}\right)+d\left(\gamma^{\prime}\right)}\left|\Phi\left(\gamma^{\prime}\right)\right| \leqq a(\gamma)
$$

for each $\gamma \in K$. Then $\mathscr{Z}(\mathrm{L} ; \Phi) \neq 0$ for each finite $L \subset K$ and there exists a unique function $\Phi^{T}: \mathscr{B} \rightarrow \mathbb{C}$ such that

for every $\mathrm{L} \in \mathscr{B}$.

$$
\log \mathscr{Z}(\mathrm{L} ; \Phi)=\sum_{\mathrm{C}: \mathrm{C} \subset \mathrm{L}} \Phi^{T}(\mathrm{C})
$$

Moreover the function $\Phi^{T}$ is given by the formula

$$
\Phi^{T}(\mathrm{C})=\sum_{\mathrm{B}: \mathrm{B} \subset \mathrm{C}}(-1)^{|\mathrm{C}|-|\mathrm{B}|} \log \mathscr{Z}(\mathrm{B} ; \Phi),
$$

the estimate

$$
\sum_{\substack{\mathrm{C} \in \mathscr{R} \\ \mathrm{C} \iota \gamma}}\left|\Phi^{T}(\mathrm{C})\right| e^{d(\mathrm{C})} \leqq a(\gamma)
$$


holds true for every $\gamma \in \mathrm{K}$, and

$$
\Phi^{T}(\mathrm{C})=0 \quad \text { whenever } \mathrm{C} \text { is not a cluster. }
$$

The proof is given in Sect. 3 .

Let us mention that the use of Möbius inversion (3) goes back to e.g. [8] (see also [1]) and was later advocated by Mack [9]. He also suggested that the formulas like (2) and (3) might be used as a computational tool-computing from (3) the first few relevant terms in the expansion (2). Assumptions of the type (1) were used by Guerra [10], in slightly stronger form, who applied it in a proof of the existence of infinite volume limits of correlations, and by Navrátil [11], who derived (4) from (1) applying the Kirkwood-Salsburg equation approach according to [5].

To evaluate the strength of the assumption (1) let us compare it with the recent paper of Cammarota [7] (see also [6]). To do so, let us suppose that polymers are certain geometrical objects and that the number of all polymers containing a given lattice site and of the length $|\gamma|=n$ may be bounded by $K^{n}$, where $K$ is certain constant. Then Cammarota proves the convergence of the cluster expansion if $|\Phi(\gamma)| \leqq e^{-\tau|\gamma|}$ with $\tau>\log K+\log 5=\log K+1,609 \ldots$ Assuming that $d=0$ and $a(\gamma)=a|\gamma|$, we get (1) if

$$
\sum_{n=1}^{\infty}\left(K e^{a-\tau}\right)^{n}=\frac{e^{a-\tau+\log K}}{1-e^{a-\tau+\log K}} \leqq a .
$$
One easily verifies that the most convenient choice of $a$ is $a=\frac{\sqrt{5}-1}{2}$ yielding the
slightly better estimate

$$
\tau>\log K+a+\log (1+a)-\log a=\log K+1,580 \ldots .
$$

Formula (2) together with estimate (4) yield a convenient description for polymer models. Whenever polymers are geometrical objects on some lattice, one may write down a closed form expression for the bulk free energy and one has a good control of boundary terms when comparing it with the finite volume partition function. Without going into details, let us mention that if $\|\mathrm{C}\|$ denotes the number of sites of the underlying lattice in all polymers from $\mathrm{C}$, we get for the translation invariant case

$$
\lim \frac{1}{|V|} \log Z(V)=\sum_{\mathrm{C}: \operatorname{supp} \mathrm{C}_{\ni i}} \frac{\Phi^{T}(\mathrm{C})}{\|\mathrm{C}\|}
$$

with the sum over all clusters containing a fixed site $i$ in some of their polymers. Let us remark also that whenever $\Phi$ is analytic in some parameter, then clearly both $Z(V)$ and $\Phi^{T}(C)$ are analytic, and thus the estimate (4) and Vitali theorem imply the analyticity of the free energy and similar things.

Formulas (2) and (4) are also useful for an evaluation of the decay rate of correlations. This may be done already in our abstract setting. Let us introduce the correlations

$$
\varrho_{\mathrm{L}}(\partial ; \Phi)=\chi_{\mathrm{L}}(\partial) \cdot \frac{\sum_{\bar{\partial} \in \mathscr{Q}(\mathrm{L}), \bar{\partial} \partial} \Phi(\partial)}{\mathscr{Z}(\mathrm{L} ; \Phi)}
$$


whenever $\partial \in \mathscr{D}_{0}$ and $L \subset K$ is finite. Here $\chi_{\mathrm{L}}(\partial)$ is defined to equal 1 if $\partial \subset L$ and 0 otherwise. Let us denote the set of all clusters in $L$ by $\mathscr{C}(\mathrm{L}), \mathscr{C}=\mathscr{C}(\mathrm{K})$, and define

$$
d(\partial, A)=\inf \{d(C) \mid C \in \mathscr{C}, C l \partial, C \cap A \neq \emptyset\}
$$

whenever $\partial \in \mathscr{D}_{0}$ and $A \subset K$,

$$
d\left(\partial_{1}, \partial_{2}\right)=\inf \left\{d(C) \mid C \in \mathscr{C}, \mathrm{C}_{l} \partial_{1}, \mathrm{C}_{l} \partial_{2}\right\}
$$

whenever $\partial_{1}, \partial_{2} \in \mathscr{D}_{0}$, and finally

$$
a(\partial)=\sum_{\gamma \in \delta} a(\gamma) \text { for } \partial \in \mathscr{D}_{0} .
$$

Standard statements about the decay of correlations (cf. e.g. [1-4]) may be summarized in the following

Proposition. Let $\Phi$ be a polymer functional and suppose that there exists a function $\Phi^{T}: \mathscr{C} \rightarrow \mathbb{C}$ such that

$$
\mathscr{Z}(\mathrm{L} ; \Phi)=\exp \left[\sum_{\mathrm{C} \in \mathscr{C}(\mathrm{L})} \Phi^{T}(\mathrm{C})\right]
$$

for every finite $\mathrm{LCK}$ and a function $d: \mathrm{K} \rightarrow[0, \infty)$ such that

$$
\sum_{\substack{\mathrm{C} \in \mathscr{C} \\ \mathrm{C} l \gamma}}\left|\Phi^{T}(\mathrm{C})\right| e^{d(\mathrm{C})} \leqq a(\gamma)
$$

for every $\gamma \in \mathrm{K}$ and some function $a: \mathrm{K} \rightarrow[0, \infty)$. Then for every $\partial \in \mathscr{D}_{0}$ and every $\mathrm{L}, \mathrm{L}_{1}, \mathrm{~L}_{2} \in \mathscr{B}$ one has

(i) $\left|\varrho_{\mathrm{L}}(\partial ; \Phi)\right| \leqq \chi_{\mathrm{L}}(\partial) e^{a(\partial)}|\Phi(\partial)|$,

(ii) $\lim _{\mathrm{L} \rightarrow \mathrm{K}} \varrho_{\mathrm{L}}(\partial ; \Phi)=\Phi(\partial) \exp \left[\sum_{\substack{\mathrm{C} \in \mathscr{C} \\ \mathrm{C}_{1} \partial}} \Phi^{T}(\mathrm{C})\right]$

(iii) $\left|\varrho_{\mathrm{L}_{1}}(\partial ; \Phi)-\varrho_{\mathrm{L}_{2}}(\partial ; \Phi)\right| \leqq|\Phi(\partial)| a(\partial) e^{a(\partial)} e^{-d\left(\partial, \mathrm{L}_{1} \div \mathrm{L}_{2}\right)}$, where $\mathrm{L}_{1} \div \mathrm{L}_{2}=\left(\mathrm{L}_{1} \backslash \mathrm{L}_{2}\right)$ $\cup\left(L_{2} \backslash L_{1}\right)$,

(iv) $\left|\varrho_{\mathrm{L}}\left(\partial_{1} \cup \partial_{2} ; \Phi\right)-\varrho_{\mathrm{L}}\left(\partial_{1} ; \Phi\right) \varrho_{\mathrm{L}}\left(\partial_{2} ; \Phi\right)\right|$ whenever $\partial_{1} \cup \partial_{2} \in \mathscr{D}_{0}$.

$$
\leqq \chi_{\mathrm{L}}\left(\partial_{1} \cup \partial_{2}\right)\left|\Phi\left(\partial_{1} \cup \partial_{2}\right)\right| a\left(\partial_{1} \cup \partial_{2}\right) e^{a\left(\partial_{1} \cup \partial_{2}\right)-d\left(\partial_{1} \cup \partial_{2}\right)}
$$

Proof. In view of $\left(2^{\prime}\right)$ and denoting $[\partial]=\{\gamma \in K \mid \gamma l \partial\}$, we have

$$
\varrho_{L}(\partial ; \Phi)=\chi_{\mathrm{L}}(\partial) \Phi(\partial) \frac{\mathscr{Z}(\mathrm{L} \backslash[\partial])}{\mathscr{Z}(\mathrm{L})}=\chi_{\mathrm{L}}(\partial) \Phi(\partial) \exp \left[-\sum_{\substack{\mathrm{C} \in \mathscr{C}(\mathrm{L}) \\ \mathrm{C} / \partial}} \Phi^{T}(\mathrm{C})\right] .
$$

Both (i) and (ii) then immediately follow from (4). To prove (iii) and (iv) one uses (5), the inequality

$$
\left|e^{z_{1}}-e^{z_{2}}\right| \leqq \max \left(e^{\left|z_{1}\right|}, e^{\left|z_{2}\right|}\right)\left|z_{1}-z_{2}\right| \quad \text { valid for every } z_{1}, z_{2} \in \mathbb{C},
$$

and the inequalities

$$
\sum_{\substack{\mathrm{C} \in \mathscr{C} \\ \mathrm{C}_{l} \partial, \mathrm{C} \cap \mathrm{L}_{1} \div \mathrm{L}_{2} \neq 0}}\left|\Phi^{T}(\mathrm{C})\right| \leqq e^{-d\left(\partial, \mathrm{L}_{1} \div \mathrm{L}_{2}\right)} \sum_{\substack{\mathrm{C} \in \mathscr{C} \\ \mathrm{C}_{l} \partial}}\left|\Phi^{T}(\mathrm{C})\right| e^{d(\mathrm{C})}
$$

for (iii) and

for (iv).

$$
\sum_{\substack{\mathrm{C} \in \mathscr{C} \\ \mathrm{C} \imath \partial_{1} \text { and } \mathrm{C}_{l} \partial_{2}}}\left|\Phi^{T}(\mathrm{C})\right| \leqq e^{-d\left(\partial_{1}, \partial_{2}\right)} \sum_{\substack{\mathrm{C} \in \mathscr{C} \\ \mathrm{C}_{\imath}\left(\partial_{1} \cup \partial_{2}\right)}}\left|\Phi^{T}(\mathrm{C})\right| e^{d(\mathrm{C})}
$$


Let us finally remark that, in analogy with (3), one may introduce functions $\Delta_{\partial}: \mathscr{B} \rightarrow \mathbb{C}$ for every $\partial \in \mathscr{D}_{0}$ by

$$
\Delta_{\partial}(C)=\sum_{B \subset C}(-1)^{|C|-|B|} \varrho_{B}(\partial)
$$

and to prove in a straightforward way that

$$
\varrho_{\mathrm{L}}(\partial)=\sum_{\mathrm{C} \subset \mathrm{L}} \Delta_{\partial}(\mathrm{C})
$$

for each $L \in \mathscr{B}$ and that

$$
\sum_{C \in \mathscr{B}}\left|\Delta_{\partial}(C)\right| e^{d(\mathcal{C})} \leqq e^{a(\partial)+d(\partial)}|\Phi(\partial)| .
$$

Moreover $\Delta_{\partial}(\mathrm{C})$ factorizes: $\Delta_{\partial}(\mathrm{C})=\Delta_{\partial_{1}}\left(\mathrm{C}_{1}\right) \cdot \Delta_{\partial_{2}}\left(\mathrm{C}_{2}\right)$ whenever $\partial=\partial_{1} \cup \partial_{2}$, $\mathrm{C}=\mathrm{C}_{1} \cup \mathrm{C}_{2}$ and every $\gamma \in \partial_{1} \cup \mathrm{C}_{1}$ is compatible with every $\gamma^{\prime} \in \partial_{2} \cup \mathrm{C}_{2}$. Using the estimate (8) one may slightly improve (iii) and (iv) by omitting the factor $a(\partial)$, respectively $a\left(\partial_{1} \cup \partial_{2}\right)$ on the right-hand side. Let us note also that if $\Phi(\gamma)$ is nonnegative for each $\gamma \in \mathrm{K}$, the estimates (i), (iii), and (iv) may be improved by skipping $e^{a(\partial)}$, respectively $e^{a\left(\partial_{1} \cup \partial_{2}\right)}$. But in this case already the theorem may be considerably improved [12].

\section{Proof of Theorem}

To prove the unicity of $\Phi^{T}$, suppose that $\mathscr{Z}(\mathrm{L} ; \Phi) \neq 0$ whenever $\mathrm{L} \in \mathscr{B}$. If (2) is satisfied, then

$$
\begin{gathered}
\sum_{\mathrm{B}: \mathrm{B} C \mathrm{C}}(-1)^{|\mathrm{C}|-|\mathrm{B}|} \log \mathscr{Z}(\mathrm{B} ; \Phi)=\sum_{\mathrm{B}: \mathrm{B} C \mathrm{C}}(-1)^{|\mathrm{C}|-|\mathrm{B}|} \sum_{\mathrm{A}: \mathrm{A} \subset \mathrm{C}} \Phi^{T}(\mathrm{~A}) \\
=\sum_{\mathrm{A}: \mathrm{A} \subset \mathrm{C}} \Phi^{T}(\mathrm{~A}) \sum_{\mathrm{B}: \mathrm{A} \subset \mathrm{B} C \mathrm{C}}(-1)^{|\mathrm{C}|-|\mathrm{B}|}=\Phi^{T}(\mathrm{C}),
\end{gathered}
$$

since

$$
\sum_{B: A \subset B C C}(-1)^{|C|-|B|}=\sum_{D \subset C \backslash A}(-1)^{|C|-|A|-|D|}=(1-1)^{|C| A \mid}=0
$$

whenever $A \neq C$. This proves the unicity of $\Phi^{T}$. Similarly one easily shows that (3) implies (2) and also the fact that $\Phi^{T}(\mathrm{C})$ vanishes whenever $\mathrm{C}$ is not a cluster.

Realizing that the expression (3) for $\Phi^{T}(\mathrm{C})$ uses only $\mathscr{Z}(\mathrm{B} ; \Phi)$ with $\mathrm{B} \subset \mathrm{C}$, the proof will be finished after verifying the following statement for every finite $L C K$ :

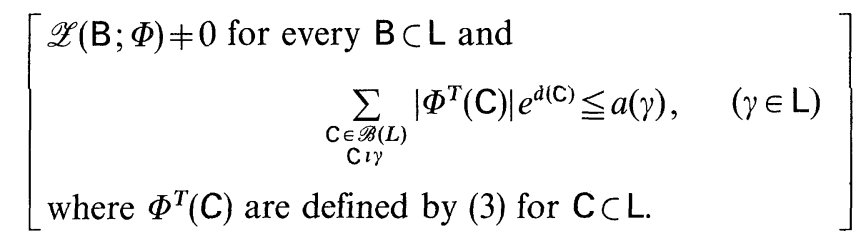

Let us fix a finite LCK and denote by $\left(1_{s}\right)$ the modified assumption (1)

$$
\sum_{\gamma^{\prime}: \gamma^{\prime}, \gamma} e^{a\left(\gamma^{\prime}\right)+d\left(\gamma^{\prime}\right)}\left|\Phi\left(\gamma^{\prime}\right)\right| \leqq s \cdot a(\gamma)
$$


and consider the set $I$ of those $s \in[0,1]$ for which $(S)$ holds true for every $\Phi$ fulfilling $\left(1_{s}\right)$. The set $I$ is nonempty since $0 \in I$ [the only functional fulfilling $\left(1_{0}\right)$ being the zero functional $]$ and thus $\alpha=\sup I \in[0,1]$.

We shall prove first that $\alpha \in I$. In fact, let $\Phi$ fulfill $\left(1_{\alpha}\right)$. The functional $\Phi_{t}=t \Phi$ satisfies $\left(1_{t \alpha}\right)$ and thus also $(S)$ for every $t \in[0,1)$. Hence for every $t \in[0,1)$ and every $B C L$ we have

$$
\left|\mathscr{Z}\left(\mathrm{B} ; \Phi_{t}\right)\right|=\mid \exp \left[\sum_{\mathrm{C} \subset \mathrm{B}} \Phi_{t}^{T}(\mathrm{C})\right] \geqq \exp \left[-\sum_{\gamma \in L} a(\gamma)\right],
$$

since

$$
\left|\sum_{\mathrm{C} \subset \mathrm{B}} \Phi_{t}^{T}(\mathrm{C})\right| \leqq \sum_{\gamma \in \mathrm{L}} \sum_{C \gamma \gamma}\left|\Phi_{t}^{T}(\mathrm{C})\right| \leqq \sum_{\gamma \in \mathrm{L}} a(\gamma) .
$$

$\mathscr{Z}\left(\mathrm{B} ; \Phi_{t}\right)$ is continuous as a function of $t$ and thus it follows from (9) that $\mathscr{Z}(\mathrm{B} ; \Phi) \neq 0$. Hence we may define $\Phi^{T}$ by (3), and since according to (3) it is $\Phi^{T}(\mathrm{C})$ $=\lim _{t \rightarrow 1} \Phi_{t}^{T}$, the estimate in $(S)$ is fulfilled for $\Phi^{T}$.

Suppose now that $\alpha<1$. We shall show that

$$
\sum_{\substack{\mathrm{C} \in \mathscr{\mathscr { B }}(L) \\ \mathrm{C} l \gamma}}\left|\Phi^{T}(\mathrm{C})\right| e^{d(\mathrm{C})} \leqq \alpha \cdot a(\gamma)
$$

for every $\Phi$ satisfying $\left(1_{\alpha}\right)$. But this would contradict the assumption that $\alpha=\sup I$. To see it, we notice that the set of contour functionals fulfilling $\left(1_{\alpha}\right)$ is a compact space contained in the open set $\{\Phi \mid \mathscr{Z}(\mathrm{B} ; \Phi) \neq 0$ for each $\mathrm{B} C \mathrm{~L}\}$. Hence, there is $\alpha_{1}>\alpha$ such that $\mathscr{Z}(\mathrm{B} ; \Phi) \neq 0$ for every B $\subset$ L and every $\Phi$ fulfilling $\left(1_{\alpha_{1}}\right)$. Similarly, the set of all $\Phi$ fulfilling $\left(1_{\alpha_{1}}\right)$ and such that $\sum_{\substack{\mathrm{C}_{\in} \mathscr{\mathscr { B } ( L )} \\ \mathrm{C} / \gamma}}\left|\Phi^{T}(\mathrm{C})\right| e^{d(\mathrm{C})}<a(\gamma)$ whenever $\gamma \in L$ and $a(\gamma)>0$ is a relatively open subset of $\left\{\Phi \mid \Phi\right.$ fulfills $\left.\left(1_{\alpha_{1}}\right)\right\}$, and in view of (10) it contains $\left\{\Phi \mid \Phi\right.$ fulfills $\left.\left(1_{\alpha_{1}}\right)\right\}$. Thus, there exists $\alpha_{2} \in\left(\alpha, \alpha_{1}\right)$ such that the inequality from $(S)$ holds true for $a(\gamma)>0$ and $\Phi$ fulfilling $\left(1_{\alpha_{2}}\right)$. But, if $a(\gamma)=0$, then $\Phi(\gamma)=0$ for all $\gamma^{\prime} l \gamma$, and thus $\Phi^{T}(\mathrm{C})=0$ for every $C_{l} \gamma$. To verify the last fact we consider any $\gamma^{\prime} \in \mathrm{C}$ such that $\gamma^{\prime} \imath \gamma$ and use (3) to get

$$
\begin{aligned}
\Phi^{T}(\mathrm{C}) & =\sum_{\substack{\mathrm{B} C \mathrm{C} \\
B \ni \vartheta^{\prime}}}(-1)^{|\mathrm{C}|-|\mathrm{B}|} \log \mathscr{Z}(\mathrm{B} ; \Phi)+\sum_{\substack{\mathrm{B} C \mathrm{C} \\
\mathrm{B} \not \gamma^{\prime}}}(-1)^{|\mathrm{C}|-|\mathrm{B}|} \log \mathscr{L}(\mathrm{B} ; \Phi) \\
& =\sum_{\substack{\mathrm{B} C \mathrm{C} \\
\mathrm{B} \ni \gamma^{\prime}}}(-1)^{|\mathrm{C}|-|\mathrm{B}|}\left[\log \mathscr{L}(\mathrm{B} ; \Phi)-\log \mathscr{L}\left(\mathrm{B} \backslash\left\{\gamma^{\prime}\right\} ; \Phi\right)\right]=0,
\end{aligned}
$$

since $\Phi\left(\gamma^{\prime}\right)=0$ and thus $\mathscr{Z}(\mathrm{B} ; \Phi)=\mathscr{Z}\left(\mathrm{B} \backslash\left\{\gamma^{\prime}\right\} ; \Phi\right)$. Thus $(S)$ is verified for all $\Phi$ satisfying $\left(1_{\alpha_{2}}\right)$.

Finally, to prove (10) let us consider a functional $\Phi$ fulfilling $\left(1_{\alpha}\right)$ and fix $\gamma \in \mathrm{L}$. Defining now $\Phi_{t}\left(\gamma^{\prime}\right)=t \Phi\left(\gamma^{\prime}\right)$ for every $\gamma^{\prime} t \gamma$ and $\Phi_{t}\left(\gamma^{\prime}\right)=\Phi\left(\gamma^{\prime}\right)$ for remaining $\gamma^{\prime}$ and introducing $\varepsilon(\mathrm{C}) \in \mathbb{C}$ such that $\left|\Phi^{T}(\mathrm{C})\right|=\Phi^{T}(\mathrm{C}) \cdot \varepsilon(\mathrm{C})$, we get

$$
\sum_{\substack{\mathrm{C} \in \mathscr{\mathscr { B }}(\mathrm{L}) \\ \mathrm{C} i \gamma}}\left|\Phi^{T}(\mathrm{C})\right| e^{d(\mathrm{C})}=\int_{0}^{1} \frac{d}{d t}\left[\sum_{\substack{\mathrm{C} \in \mathscr{P}(\mathrm{L}) \\ \mathrm{C} l \gamma}} \Phi_{t}^{T}(\mathrm{C}) \varepsilon(\mathrm{C}) e^{d(\mathrm{C})}\right],
$$


since the same argument as that in (11) shows that $\Phi_{0}^{T}(\mathrm{C})=0$ for every $\mathrm{C} \in \mathscr{B}(\mathrm{L})$ such that $\mathrm{C}_{\imath} \gamma$. To evaluate the right-hand side of (12) we observe that $\Phi_{t}$ satisfies $\left(1_{\alpha}\right)$ and thus also $(S)$ and using this, the formula (2), the definition of $\mathscr{Z}\left(\mathrm{B} ; \Phi_{t}\right)$, and recalling that $\left[\gamma^{\prime}\right]$ is the set of all polymers incompatible with $\gamma^{\prime}$, we get

$$
\begin{aligned}
& \frac{d}{d t} \log \mathscr{Z}\left(\mathrm{B} ; \Phi_{t}\right)=\sum_{\substack{\gamma^{\prime} \in \mathrm{B} \\
\gamma^{\prime} \iota \gamma}} \frac{\partial \log \mathscr{L}\left(\mathrm{B} ; \Phi_{t}\right)}{\partial\left(\Phi_{t}\left(\gamma^{\prime}\right)\right)} \cdot \frac{d \Phi_{t}\left(\gamma^{\prime}\right)}{d t} \\
& \quad=\sum_{\substack{\gamma^{\prime} \in \mathrm{B} \\
\gamma^{\prime} \nu \gamma}} \Phi\left(\gamma^{\prime}\right) \frac{\mathscr{Z}\left(\mathrm{B} \backslash[\gamma] ; \Phi_{t}\right)}{\mathscr{Z}\left(\mathrm{B} ; \Phi_{t}\right)}=\sum_{\substack{\gamma^{\prime} \in \mathrm{B} \\
\gamma^{\prime} i \gamma}} \Phi\left(\gamma^{\prime}\right) \exp \left[-\sum_{\substack{\mathrm{D} \subset \mathrm{B} \\
\mathrm{D} i \gamma^{\prime}}} \Phi_{t}^{T}(\mathrm{D})\right] .
\end{aligned}
$$

Hence

$$
\begin{aligned}
& \frac{d}{d t}\left[\sum_{\substack{\mathrm{C} \in \mathscr{B}(L) \\
\mathrm{C} l \gamma}} \Phi_{t}^{T}(\mathrm{C}) \varepsilon(\mathrm{C}) e^{d(\mathbf{C})}\right] \\
& =\sum_{\substack{\mathrm{C} \in \mathscr{G}(L) \\
\mathrm{C} \gamma \gamma}} \varepsilon(\mathrm{C}) e^{d(\mathrm{C})} \sum_{\mathrm{B} \subset \mathrm{C}}(-1)^{|\mathrm{C}|-|\mathrm{B}|} \frac{d}{d t} \log \mathscr{Z}\left(\mathrm{B} ; \Phi_{t}\right) \\
& =\sum_{\substack{\gamma^{\prime} \in \mathrm{L} \\
\gamma^{\prime} L \gamma}} \Phi\left(\gamma^{\prime}\right) \sum_{\substack{\mathrm{C} \in \mathscr{\mathscr { O }}(\mathrm{L}) \\
\mathrm{C} \ni \gamma^{\prime}}} \varepsilon(\mathrm{C}) e^{d(\mathrm{C})} \sum_{\substack{\mathrm{B} C \mathrm{C} \\
\mathrm{B} \exists \gamma^{\prime}}}(-1)^{|\mathrm{C}|-|\mathrm{B}|} \exp \left[-\sum_{\substack{\mathrm{D} C \mathrm{~B} \\
\mathrm{D} i \gamma^{\prime}}} \Phi_{t}^{T}(\mathrm{D})\right] \\
& =\sum_{\substack{\gamma^{\prime} \in \mathbf{L} \\
\gamma^{\prime}, \gamma}} \Phi\left(\gamma^{\prime}\right) \sum_{\substack{\mathrm{C} \in \mathscr{G}(L) \\
\mathrm{C} \ni \gamma^{\prime}}} \varepsilon(\mathrm{C}) e^{d(\mathrm{C})} \sum_{n=0}^{\infty} \frac{1}{n !} \\
& \cdot \sum_{\substack{D_{1}, \ldots, D_{n} \subset L \\
D_{1} 1 \gamma^{\prime}, \ldots, D_{n} \gamma^{\prime}}} \prod_{\ell=1}^{n}\left(-\Phi_{t}^{T}\left(D_{\ell}\right)\right) \sum_{\substack{B \subset C \\
B \supset \cup D_{\ell} \cup\left\{\gamma^{\prime}\right\}}}(-1)^{|C|-|B|} \\
& =\sum_{\substack{\gamma^{\prime} \in \mathrm{L} \\
\gamma^{\prime} l \gamma}} \Phi\left(\gamma^{\prime}\right) \sum_{n=0} \frac{1}{n !} \sum_{\substack{\mathrm{D}_{1}, \ldots, \mathrm{D}_{n} \subset L \\
\mathrm{D}_{1} \gamma^{\prime}, \ldots, \mathrm{D}_{n} l \gamma^{\prime}}} \prod_{\ell=1}^{n}\left(-\Phi_{t}^{T}\left(\mathrm{D}_{\ell}\right)\right) \varepsilon\left(\cup \mathrm{D}_{\ell} \cup\left\{\gamma^{\prime}\right\}\right) e^{d\left(\cup \mathrm{D}_{\ell} \cup\left\{\gamma^{\prime}\right\}\right)} .
\end{aligned}
$$

In the last equality we used the fact that $\sum_{\substack{B \subset C \\ B \supset \cup D_{\ell} \cup\left\{y^{\prime}\right\}}}(-1)^{|C|-|B|}=0$ whenever $\cup \mathrm{D}_{\ell} \cup\left\{\gamma^{\prime}\right\} \neq \mathrm{C}$. Thus recalling that $d\left(\cup \mathrm{D}_{\ell} \cup\left\{\gamma^{\prime}\right\}\right) \leqq \sum d\left(\mathrm{D}_{\ell}\right)+d\left(\gamma^{\prime}\right)$, and using $(S)$ for $\Phi_{t}$ and $\left(1_{\alpha}\right)$ for $\Phi$, we get

$$
\begin{aligned}
& \left|\frac{d}{d t}\left[\sum_{\substack{\mathrm{C} \in \mathscr{B}(\mathrm{L}) \\
\mathrm{C} \nu \gamma}} \Phi_{t}^{T}(\mathrm{C}) \varepsilon(\mathrm{C}) e^{d(\mathrm{C})}\right]\right| \\
& \leqq \sum_{\substack{\gamma^{\prime} \in L \\
\gamma^{\prime} L \gamma}}\left|\Phi\left(\gamma^{\prime}\right)\right| e^{d\left(\gamma^{\prime}\right)} \sum_{n=0} \frac{1}{n !} \sum_{\substack{\mathrm{D}_{1}, \ldots, \mathrm{D}_{n} \subset L \\
\mathrm{D}_{1}\left(\gamma^{\prime}, \ldots, \mathrm{D}_{n} \gamma^{\prime} \gamma^{\prime}\right.}} \prod_{\ell=1}^{n}\left(\left|\Phi_{t}^{T}\left(\mathrm{D}_{t}\right)\right| e^{d\left(\mathrm{D}_{\ell}\right)}\right) \\
& =\sum_{\substack{\gamma^{\prime} \in \mathrm{L} \\
\gamma^{\prime} \imath \gamma}}|\Phi(\gamma)| e^{d\left(\gamma^{\prime}\right)} \exp \left[\sum_{\substack{\mathrm{D} \subset L \\
\mathrm{D} \ell \gamma^{\prime}}}\left|\Phi_{t}^{T}(\mathrm{D})\right| e^{d(\mathrm{D})}\right] \\
& \leqq \sum_{\substack{\gamma^{\prime} \in \mathrm{L} \\
\gamma^{\prime} \imath \gamma}}\left|\Phi\left(\gamma^{\prime}\right)\right| e^{d\left(\gamma^{\prime}\right)+a\left(\gamma^{\prime}\right)} \leqq \alpha a(\gamma) .
\end{aligned}
$$

Using this in (12) we finally get (10). 


\section{References}

1. Malyshev, V.A.: Cluster expansions in lattice models in statistical physics and quantum field theory. Usp. Mat. Nauk 35, 3-53 (1980)

2. Ruelle, D.: Statistical mechanics: Rigorous results. New York: Benjamin 1969

3. Minlos, R.A., Sinai, Ya.G.: The phenomenon of "phase separation" at low temperatures in some lattice models of a gas. I, II. Math. USSR-Sb. 2, 335-395 (1967) and Trans. Mosc. Math. Soc. 19, 121-196 (1968)

Sinai, Ya.G.: Theory of phase transitions: rigorous results. Budapest and London: Akadémiai Kiadó and Pergamon Press 1982

4. Gruber, C., Kunz, H.: General properties of polymer systems. Commun. Math. Phys. 22, 133-161 (1971)

5. Gallavotti, G., Martin-Löf, A., Miracle-Sole, S.: Some problems connected with the description of coexisting phases at low temperatures in the Ising model. Battelle Seattle 1971 Recontres. A. Lenard, (ed.). Lecture Notes in Physics, Vol. 20, pp. 162-204. Berlin, Heidelberg, New York: Springer 1973

6. Seiler, E.: Gauge theories as a problem of constructive quantum field theory and statistical mechanics. Lecture Notes in Physics, Vol. 159. Berlin, Heidelberg, New York: Springer 1982

7. Cammarota, C.: Decay of correlations for infinite range interactions in unbounded spin systems. Commun. Math. Phys. 85, 517 (1982)

8. Rushbrooke, G.S., Baker, G.A., Wood, P.J.: Heisenberg model, pp. 245-356. Domb, C.: Graph theory and embeddings, pp. 1-95. In:Phase transitions and critical phenomena, Vol. 3. Domb, C., Green, M.S. (eds.). London, New York, San Francisco: Academic Press 1974

9. Mack, G.: Nonperturbative methods. In: Gange theories of the eighties. Raitio, R., Lindfors, J. (eds.). Lecture Notes in Physics, Vol. 181. Berlin, Heidelberg, New York: Springer 1983

10. Guerra, F.: Gauge fields on a lattice; selected topics. In: Field theoretical methods in particle physics, pp. 41-65. Rühl, W. (ed.). New York, London: Plenum Press 1980

11. Navrátil, J.: Contour models and unicity of random fields. (In Czech). Diploma thesis, Charles University, Prague (1982)

12. Kotecký, R., Preiss, D.: Dobrushin unicity and its application to polymer models (in preparation)

Communicated by J. Fröhlich

Received July 1, 1985 